\title{
Resonant Instability of Kink Oscillations in Magnetic Flux Tubes with Siphon Flow
}

\author{
Michael S. Ruderman ${ }^{1,2,3}$ (D) Nikolai S. Petrukhin ${ }^{4}$
}

Received: 22 February 2021 / Accepted: 26 May 2021 / Published online: 29 June 2021

(c) The Author(s) 2021

\begin{abstract}
We study kink oscillations of a straight magnetic tube in the presence of siphon flows. The tube consists of a core and a transitional or boundary layer. The flow velocity is parallel to the tube axis, has constant magnitude, and confined in the tube core. The plasma density is constant in the tube core and it monotonically decreases in the transitional layer to its value in the surrounding plasma. We use the expression for the decrement/increment previously obtained by Ruderman and Petrukhin (Astron. Astrophys. 631, A31, 2019) to study the damping and resonant instability of kink oscillations. We show that, depending on the magnitude of siphon-velocity, resonant absorption can cause either the damping of kink oscillations or their enhancement. There are two threshold velocities: When the flow velocity is below the first threshold velocity, kink oscillations damp. When the flow velocity is above the second threshold velocity, the kink oscillation amplitudes grow. Finally, when the flow velocity is between the two threshold velocities, the oscillation amplitudes do not change. We apply the theoretical result to kink oscillations of prominence threads. We show that, for particular values of thread parameters, resonant instability can excite these kink oscillations.
\end{abstract}

Keywords Sun · Plasma $\cdot$ Magnetohydrodynamics · Waves · Oscillations · Stability

\section{Introduction}

Kink oscillations of coronal magnetic tubes were first observed by the Transition Region and Coronal Explorer (TRACE) mission in 1998. These observations were reported by Aschwanden et al. (1999) and Nakariakov et al. (1999). Now these oscillations are routinely

This article belongs to the Topical Collection:

Magnetohydrodynamic (MHD) Waves and Oscillations in the Sun's Corona and MHD Coronal

Seismology

Guest Editors: Dmitrii Kolotkov and Bo Li

M.S. Ruderman

m.s.ruderman@sheffield.ac.uk

1 Solar Physics and Space Plasma Research Centre (SP2RC), School of Mathematics and Statistics, University of Sheffield, Hicks Building, Hounsfield Road, Sheffield, S3 7RH, UK

2 Space Research Institute (IKI) Russian Academy of Sciences, Moscow, Russia

3 Moscow Center for Fundamental and Applied Mathematics, Lomonosov Moscow State University, Moscow, Russia

4 National Research University - Higher School of Economics, Moscow, Russia 
observed by space missions (e.g. Erdélyi and Taroyan, 2008; Duckenfield et al., 2018; Su et al., 2018; Abedini, 2018, and the references therein). Kink oscillations were also observed in prominence threads (e.g. Arregui, Oliver, and Ballester, 2018).

Flows are ubiquitously present in magnetic structures in the solar atmosphere. In particular, they were observed in active-region loops by the Solar and Heliospheric Observatory (SOHO) (Brekke, Kjeldseth-Moe, and Harrison, 1997; Teriaca et al., 2004), TRACE (Winebarger, DeLuca, and Golub, 2001; Winebarger et al., 2002; Doyle et al., 2006), and more recently by Hinode (Teriaca et al., 2004; Tian et al., 2008; Ofman and Wang, 2008), and the Solar Terrestrial Relations Observatory (STEREO) (Tian et al., 2009). Flows with the speed between about $5 \mathrm{~km} \mathrm{~s}^{-1}$ and $30 \mathrm{~km} \mathrm{~s}^{-1}$ were also often observed in prominence threads (Chae et al., 2008; Terradas et al., 2008).

A few mechanisms of this have been considered. Nakariakov et al. (1999) suggested that the kink oscillations of coronal magnetic loops are excited by nearby solar flares. It was confirmed by the statistical analysis by Zimovets and Nakariakov (2015) that the majority of coronal-loop kink oscillations are excited by this mechanism. For some time this was the only mechanism suggested by theorists. Later two other mechanisms were proposed: The first one is the Alfvénic vortex shedding suggested by Gruszecki et al. (2010). The second mechanism is the excitation of kink oscillations by coronal rain studied by Kohutova and Verwichte (2017). Mechanisms of excitation of the prominence-thread kink oscillations are not very well known.

One more possible mechanism of excitation of kink waves in coronal magnetic tubes is the instability caused by the presence of a flow. The excitation of propagating kink waves by the Kelvin-Helmholtz (KH) instability has been studied intensively (e.g. Zhelyazkov, 2012; Zhelyazkov and Zaqarashvili, 2012; Zaqarashvili, Vörös, and Zhelyazkov, 2014; Zhelyazkov et al., 2015). It was found that magnetic-flux tubes with flows can be subject to the KH instability for the observed values of the flow speed, but the unstable modes are propagating fluting waves with relatively high azimuthal wave numbers. To make propagating kink waves $\mathrm{KH}$ unstable, flow speeds greatly exceeding the observed ones are needed.

To our knowledge the excitation of standing kink waves in magnetic-flux tubes in the solar corona by the $\mathrm{KH}$ instability has not been studied. However, we can use the results obtained by Ruderman (2010) who studied the effect of siphon flow on kink oscillations of thin magnetic-flux tubes. He found that the tube is $\mathrm{KH}$ unstable with respect to kink oscillations when the flow magnitude exceeds $V_{\mathrm{Ai}}\left[2\left(1+\rho_{\mathrm{i}} / \rho_{\mathrm{e}}\right)\right]^{1 / 2}$, where $V_{\mathrm{Ai}}$ is the Alfvén speed inside the tube, and $\rho_{\mathrm{i}}$ and $\rho_{\mathrm{e}}$ are the plasma densities inside and outside the tube. Then, for typical values of the coronal loops, we see that to excite kink oscillations in loops by the KH instability we need a siphon speed of the order of or larger than $1000 \mathrm{~km} \mathrm{~s}^{-1}$. The same estimate is valid for prominence threads. Hence, we should reject the KH instability as a possible mechanism of excitation of kink oscillations in coronal magnetic tubes.

However, there is another instability mechanism that can excite kink waves in magneticflux tubes. This is the Negative Energy (NE) wave instability. The concept of NE waves was first introduced by Chua in 1951 (Pierce, 1974) in application to electron beams. Then it became very popular in plasma physics (e.g. Kadomtsev, Mikhailovskii, and Timofeev, 1965; Mikhailovskii, 1974; Nezlin, 1976). In hydrodynamics the concept of negative-energy waves was first used by Benjamin (1963). However, it became popular in hydrodynamics much later when Cairns (1979) applied it to the stability of shear flows. Reviews of the theory of negative-energy waves in hydrodynamics have been given by Ostrovskii, Rybak, and Tsimring (1986) and Stepanyants and Fabrikant (1989) (see also Fabrikant and Stepanyants, 1998). To our knowledge, Ryutova (1988) was the first who applied the concept of $\mathrm{NE}$ waves to magnetohydrodynamic (MHD) waves. She studied kink waves in thin magnetic 
tubes in the presence of a homogeneous flow outside the tube and considered the implication of the obtained results to space physics. After that the application of theory of negativeenergy waves to problems of space physics was considered by many authors (e.g. Joarder, Nakariakov, and Poberts, 1997; Ruderman and Wright, 1998; Andries and Goossens, 2001; Erdélyi and Taroyan, 2003; see also reviews by Ruderman and Belov, 2010 and Taroyan and Ruderman, 2011). The main property of the NE instability is that its threshold velocity is substantially smaller than that of the KH instability.

The first observation of standing kink waves revealed that they are strongly damped, with the damping time of the order of a few oscillation periods (Nakariakov et al., 1999). Later observations showed that this property of coronal-loop kink oscillations is ubiquitous (e.g. Nechaeva et al., 2019). Kink oscillations of prominence threads also quickly damp (e.g. Arregui, Oliver, and Ballester, 2018). At present it is almost generally accepted in the solar physics that this damping is caused by resonance absorption. After Ionson (1978) pointed out the importance of resonance absorption for physical processes in the solar atmosphere, it remains a popular mechanism for explaining various solar phenomena, especially wave damping. Hollweg and Yang (1988) studied resonant damping of surface waves on a thin transitional layer sandwiched between two semi-infinite regions with cold homogeneous plasmas and constant magnetic field. Considering the limiting case of surface waves propagating almost perpendicular to the magnetic field, they managed to obtain the expression for the damping rate of kink waves propagating in a thin magnetic-flux tube. Goossens, Hollweg, and Sakurai (1992) derived the general expression for the decrement of kink waves propagating in a twisted magnetic tube. After Ruderman and Roberts (2002) and Goossens, Andries, and Aschwanden (2002) showed that resonant absorption not only adequately explains the observed damping of coronal-loop kink oscillations but also can be used to obtain seismological information about the transverse structure of coronal loops, this mechanism became especially popular in solar physics (see, e.g., Goossens, Erdélyi, and Ruderman, 2011).

When there is a negative-energy wave in a system related to the presence of flow then, as we have already mentioned, this wave becomes unstable if there is a mechanism causing the decrease of the wave energy. One such mechanism is resonant absorption. When it provides the energy sink that results in the instability of a wave, the corresponding instability is called the resonant instability. The application of resonant instability to solar physics is sparse. Andries, Tirry, and Goossens (2000) and Andries and Goossens (2001) studied the resonant instability of the magnetic slab with flow in the application to coronal plumes. Recently Bahari, Petrukhin, and Ruderman (2020) studied the resonant instability of propagating kink waves in magnetic tubes with flow with the application to waves in spicules and filaments in the solar atmosphere.

Until recently the theory of NE instability has dealt with propagating waves. Ruderman (2018) studied standing NE waves on the surface of a tangential MHD discontinuity. He used the equation derived by Ruderman and Goossens (1995) that describes surface waves on the surface of such a discontinuity in the presence of flow and fluid viscosity. This article aims to extend his analysis to kink waves in magnetic-flux tube and study their resonant instability, which is a particular case of NE instability. The article is organised as follows: In the next section we formulate the problem. In Section 3 we present the expression for the decrement/increment of kink oscillations. In Section 4 we study the resonant damping and instability of kink oscillations. In Section 5 we apply theoretical results to the problem of excitation of kink oscillations of prominence threads. Section 6 contains the summary of results and our conclusions. 


\section{Problem Formulation and Governing Equations}

We consider the plasma motion in the zero- $\beta$ plasma approximation. The unperturbed magnetic field is straight. In cylindrical coordinates $(r, \phi, z)$ it is given by $\boldsymbol{B}=B \hat{\boldsymbol{e}}_{z}$, where $\hat{\boldsymbol{e}}_{z}$ is the unit vector in the $z$-direction and $B$ is a constant. In the unperturbed state there is also a plasma flow with the velocity $\boldsymbol{U}=U(r) \hat{\boldsymbol{e}}_{z}$. The equilibrium density is given by

$$
\rho(r)=\left\{\begin{array}{cc}
\rho_{\mathrm{i}}, & r \leq R(1-\ell / 2), \\
\rho_{\mathrm{t}}(r), R(1-\ell / 2) \leq r \leq R(1+\ell / 2), \\
\rho_{\mathrm{e}}, & r \geq R(1+\ell / 2),
\end{array}\right.
$$

where $R$ is the tube radius, $\rho_{\mathrm{i}}$ and $\rho_{\mathrm{e}}$ are constants, $\rho_{\mathrm{e}}<\rho_{\mathrm{i}}, \rho_{\mathrm{t}}(r)$ is a monotonically decreasing function, and $\rho(r)$ is continuous at $r=R(1 \pm \ell / 2)$. The domain defined by $r \leq R(1-\ell / 2)$ is the core part of the magnetic tube, while $R(1-\ell / 2) \leq r \leq R(1+\ell / 2)$ is the transitional region. The velocity is in the $z$-direction and it is equal to $U$ in the core region $(r \leq R(1-\ell / 2))$, and zero in the transitional region and outside of the tube $(r>R(1-\ell / 2))$, where $U$ is a constant. We always can choose the direction of the $z$-axis in such a way that $U>0$. Since the magnetohydrodynamic equations are invariant with respect to the substitution $\boldsymbol{B} \rightarrow-\boldsymbol{B}$ we can assume that $B>0$. The unperturbed state that we use is almost the same as that used by Ruderman and Petrukhin (2019: Article I below). The only difference is the following: While it was assumed in Article I that the velocity magnitude monotonically decreases in the transitional layer from $U$ to zero, here we assume that it is a step function equal to $U$. Hence, the surface $r=R(1-\ell / 2)$ is a tangential discontinuity. We explain below why we decided to consider a simpler unperturbed state.

In general, there are three numbers characterising wave modes propagating in a magnetic tube: They are the azimuthal wave number $[m]$, the axial wave number $\left[k_{z}\right]$, and the number of modes in the radial direction. We consider kink oscillations, which correspond to $m=1$. We also consider only the first wave mode in the radial direction, which is the fundamental radial mode. Hence only one free parameter characterising the wave remains. This is the axial wave number $k_{z}$. Below we assume that the tube ends are frozen in a dense plasma mimicking the solar photosphere and consider standing waves. This implies that $k_{z}$ can take only discrete values, $k_{z}=\pi n / L, n=1,2, \ldots$, where $L$ is the tube length. Here $n=1$ corresponds to the fundamental mode and $n>1$ to the overtones. We assume that the tube is thin: $R \ll L$. In addition, we assume that the transition (also called boundary) layer is also thin: $\ell \ll 1$. Hence, we use the thin-tube and thin-boundary layer (TTTB) approximation. In this approximation the plasma displacement $\eta$ in the core region is independent of $r$ (e.g. Goossens et al., 2009; Ruderman, 2011; Ruderman, Shukhobodskiy, and Erdélyi, 2017). The displacement satisfies the frozen-in boundary conditions at the tube ends,

$$
\eta=0 \quad \text { at } z=0, L \text {. }
$$

\section{Expression for Decrement/Increment}

In Article I, damping of standing kink oscillations caused by resonant absorption was studied. As we have already stated, the equilibrium considered in Article I is similar to that described in the previous section. The only difference is that it was assumed that the flow speed is continuous and monotonically decreases in the transitional layer to zero, while in 
this article we assume that it is zero in this layer. Hence, the internal boundary of the transitional layer is a tangential discontinuity. The decrement/increment of standing kink oscillations was calculated. Thorough investigation of the derivation given in Article I shows that, in fact, it is also valid for the equilibrium considered in this article. The only condition that was used in Article I is the continuity of the radial plasma displacement and magneticpressure perturbation at the boundaries of the transitional layer. This condition is satisfied even when the internal boundary is a tangential discontinuity.

The second important assumption made in Article I is that $U$ is less than the Alfvén speed in the core region $V_{\mathrm{Ai}}$ defined by

$$
V_{\mathrm{Ai}}=\frac{B}{\sqrt{\mu_{0} \rho_{\mathrm{i}}}},
$$

where $\mu_{0}$ is the magnetic permeability of free space. This condition was only used in Article I to guarantee that the flow speed everywhere in the transitional region is less than the local Alfvén speed. Since in the equilibrium considered here the flow speed is zero in the core region, we can relax this condition. The kink wave is subjected to the KH instability when (e.g. Ruderman, 2010)

$$
U>V_{\mathrm{Ai}} \sqrt{2(\zeta+1)}=U_{\mathrm{KH}},
$$

where $\zeta=\rho_{\mathrm{i}} / \rho_{\mathrm{e}}$. The quantity $U_{\mathrm{KH}}$ is called the $\mathrm{KH}$ threshold. We only consider the KHstable kink oscillations and assume that $U<U_{\mathrm{KH}}$. However, this restriction is not sufficient. Below we impose a stronger restriction on the flow speed $U$.

It is shown in Article I that, in the leading-order approximation with respect to $\ell$, the oscillation frequency is

$$
\omega=\frac{\pi n \sqrt{\zeta}\left|2 V_{\mathrm{Ai}}^{2}-U^{2}\right|}{L \sqrt{2(\zeta+1) V_{\mathrm{Ai}}^{2}-U^{2}}} .
$$

The plasma displacement in the core region is given by

$$
\eta=\eta_{0} \mathrm{e}^{-\mathrm{i} h z} \sin \frac{\pi n z}{L}=\frac{\eta_{0}}{2 \mathrm{i}} \mathrm{e}^{-\mathrm{i} h z}\left(\mathrm{e}^{\mathrm{i} \pi n z / L}-\mathrm{e}^{-\mathrm{i} \pi n z / L}\right),
$$

where

$$
h=\frac{\omega U}{2 V_{\mathrm{Ai}}^{2}-U^{2}} .
$$

In Equations 5 and 6, $n=1$ corresponds to the fundamental mode in the $z$-direction, and $n>1$ to the $(n-1)$ th overtone. Important quantities are the Alfvén frequencies in the tube core and outside of the tube, $\left[\omega_{\mathrm{Ai}}\right.$ and $\left.\omega_{\mathrm{Ae}}\right]$, defined by

$$
\omega_{\mathrm{Ai}}=\frac{\pi V_{\mathrm{Ai}}}{L}, \quad \omega_{\mathrm{Ae}}=\frac{\pi V_{\mathrm{Ae}}}{L},
$$

where $V_{\mathrm{Ae}}^{2}=B^{2} / \mu_{0} \rho_{\mathrm{e}}$. It follows from Equation 5 that $\omega \rightarrow \infty$ as $U \rightarrow U_{\mathrm{KH}}$. Hence, for sufficiently large $U$ the wave frequency exceeds $n \omega_{\mathrm{Ae}}$ and the wave becomes leaky. Below we only consider trapped waves and impose the condition $\omega<\omega_{\mathrm{Ae}}$. It is not difficult to show that this condition is equivalent to

$$
M_{\mathrm{A}}^{2}<M_{\mathrm{L}}^{2}=\frac{3+\sqrt{8 \zeta+1}}{2},
$$


where $M_{\mathrm{A}}$ is the Alfvén Mach number calculated with respect to the Alfvén speed in the tube core. It is defined by

$$
M_{\mathrm{A}}=\frac{U}{V_{\mathrm{Ai}}} .
$$

It is easy to see that $M_{\mathrm{L}}<M_{\mathrm{KH}}=U_{\mathrm{KH}} / V_{\mathrm{Ai}}$. Hence, trapped kink waves are always $\mathrm{KH}-$ stable.

We see that $\eta$ is the superposition of two propagating waves. It is easy to show that these waves always propagate in opposite directions in the reference frame moving together with the plasma in the tube-core region. However, in the reference where the external plasma is at rest both waves propagate in the same direction when

$$
U^{2}>2 V_{\mathrm{Ai}}^{2}
$$

In this case, in accordance with the general theory the wave with smaller phase speed is a negative-energy wave.

Resonant damping or instability is related to the existence of Alfvén continuum. The Alfvén continuum $[\mathcal{V}]$ is defined by

$$
\mathcal{V}=\bigcup_{j=1}^{\infty}\left(\left[-j \omega_{\mathrm{Ae}},-j \omega_{\mathrm{Ai}}\right] \cup\left[j \omega_{\mathrm{Ai}}, j \omega_{\mathrm{Ae}}\right]\right) .
$$

A standing kink wave damps or is subjected to resonant instability only if its frequency is in the Alfvén continuum, $[\omega \in \mathcal{V}]$, otherwise the tube oscillates with constant amplitude. In the expression for $\mathcal{V}$ the two intervals with $j=1$ correspond to the Alfvén continuum related to the fundamental mode, while the two intervals with $j>0$ correspond to the Alfvén continuum related to the $(j-1)$ st overtone. Hence, the complete Alfvén continuum is a union of an infinite number of intervals.

The condition $\omega \in \mathcal{V}$ is only satisfied if there is an integer $k$ such that $k \omega \in\left[k \omega_{\mathrm{Ai}}, k \omega_{\mathrm{Ae}}\right]$. Since we assume that $\omega<\omega_{\mathrm{Ae}}$, this condition is equivalent to $\omega>k \omega_{\mathrm{Ai}}$. The intervals constituting the Alfvén continuum can overlap. Hence, the condition $\omega>k \omega_{\mathrm{Ai}}$ can be satisfied for a few values of $k$. Let $\omega>k \omega_{\mathrm{Ai}}$ for $k=1,2, \ldots, k_{1}$. If $\omega>k \omega_{\mathrm{Ai}}$ then there is such a position $r=r_{k}$ in the transitional layer where $\omega=k \omega_{\mathrm{A}}\left(r_{k}\right)$. The equation $r=r_{k}$ defines a resonant surface. We see that there are $k_{1}$ resonant surfaces in the transitional layer. Since the density monotonically decreases in the resonant layer, it follows that $\omega_{\mathrm{A}}(r)$ is a monotonically increasing function. Then it is obvious that $r_{k_{1}}<r_{k_{1}-1}<\cdots<r_{1}$.

All the resonant surfaces contribute in the decrement/increment $\gamma$. It is clearly seen in the expression for $\gamma$ obtained in Article I. For the particular equilibrium considered in this article this expression reduces to

$$
\begin{aligned}
\gamma & =\frac{4 \pi^{2} L^{2} \sqrt{\zeta}}{R V_{\mathrm{Ai}}^{2} \sqrt{2(\zeta+1) V_{\mathrm{Ai}}^{2}-U^{2}}} \sum_{k=1}^{k_{1}} \frac{k h W_{k} V_{\mathrm{A} k}^{2}}{\Delta_{k}} \\
& \times\left[\frac{h L C_{2}+\pi(n-k) C_{1}}{h^{2} L^{2}-\pi^{2}(n-k)^{2}}+\frac{h L C_{2}+\pi(n+k) C_{1}}{h^{2} L^{2}-\pi^{2}(n+k)^{2}}\right] \\
& \times \frac{1-(-1)^{n+k} \cos (h L)}{\left[h^{2} L^{2}-\pi^{2}(n-k)^{2}\right]\left[h^{2} L^{2}-\pi^{2}(n+k)^{2}\right]} .
\end{aligned}
$$


Here

$$
\begin{gathered}
\Delta_{k}=\frac{\pi^{2} k^{2}}{L^{2}} \frac{\mathrm{d} V_{\mathrm{A}}^{2}}{\mathrm{~d} r}, \quad W_{k}=\frac{\pi^{2} k^{2} V_{\mathrm{Ai}}^{2}}{L^{2}}-\frac{\omega^{2}}{\zeta}, \\
C_{1}=\frac{2 \pi n}{L}\left[h\left(V_{\mathrm{Ai}}^{2}-U^{2}\right)-\omega U\right], \\
C_{2}=\left(h^{2}+\frac{\pi^{2} n^{2}}{L^{2}}\right)\left(V_{\mathrm{Ai}}^{2}-U^{2}\right)-\omega(\omega+2 h U),
\end{gathered}
$$

$h$ is defined by Equation 7, and the subscript $k$ indicates that a quantity that depends on $r$ is calculated at $r=r_{k}$, and, as a result, it becomes a discrete function of $k$. The quantity $W$ is independent of $r$; however, the expression defining this quantity contains $k$, meaning that $W$ is also a discrete function of $k$. To explicitly show this we write $W_{k}$.

\section{Evaluation of $\gamma$}

Below we will only consider the fundamental mode and take $n=1$. Since the $(n-1)$ th overtone has a node at $z=L / n$ we can obtain the corresponding result for the $(n-1)$ th overtone just using the results obtained for the fundamental mode and reducing the tube length $n$ times. Using Equation 5 with $n=1$ we show that the condition that $\omega>\omega_{\mathrm{Ai}}$ is satisfied if either

$$
M_{\mathrm{A}}^{2}<M_{0}^{2}=\frac{4 \zeta-1-\sqrt{8 \zeta^{2}+1}}{2 \zeta},
$$

or

$$
M_{\mathrm{A}}^{2}>M_{1}^{2}=\frac{4 \zeta-1+\sqrt{8 \zeta^{2}+1}}{2 \zeta} .
$$

Next, $\omega>k \omega_{\mathrm{Ai}}, k \geq 2$, if

$$
M_{\mathrm{A}}^{2}>M_{k}^{2}=\frac{4 \zeta-k^{2}+k \sqrt{8 \zeta^{2}+k^{2}}}{2 \zeta} .
$$

It is easy to show both that $\left\{M_{k}\right\}$ is a monotonically increasing sequence and $M_{k} \rightarrow$ $\sqrt{2(\zeta+1)}$ as $k \rightarrow \infty$. The condition that $M_{k}<M_{L}$ reduces to $k<\sqrt{\zeta}$. Hence, for $\zeta \leq 4$ the condition given by Equation 19 cannot be satisfied for any $k \geq 2$. This implies that for $\zeta \leq 4$ there is either one resonant surface if $M_{\mathrm{A}}<M_{0}$ or $M_{\mathrm{A}}>M_{1}$, or no resonance surfaces at all. In general, for $k_{0}^{2}<\zeta \leq\left(k_{0}+1\right)^{2}$ Equation 19 can be satisfied for $k=2,3, \ldots k_{0}$. Hence, for $k_{0}^{2}<\zeta \leq\left(k_{0}+1\right)^{2}$ either there is no resonance surface at all, or there are up to $k_{0}$ resonant surfaces.

As an example, we consider the equilibrium with the linear density profile in the transitional layer defined by

$$
\rho_{\mathrm{t}}=\rho_{\mathrm{i}}\left[\frac{\zeta+1}{2 \zeta}+\frac{(\zeta-1)(R-r)}{\ell R \zeta}\right]
$$




\subsection{Resonant Damping of Kink Oscillations}

In this section we assume that $M_{\mathrm{A}}<M_{0}$ and study the wave damping. As was shown above, in this case there is exactly one resonant surface and we have $k_{1}=1$. The position of this resonant surface is defined by

$$
\omega_{\mathrm{A}}\left(r_{1}\right)=\omega, \quad \omega_{\mathrm{A}}(r)=\frac{\pi V_{\mathrm{A}}(r)}{L}=\frac{\pi B}{L \sqrt{\mu_{0} \rho(r)}} .
$$

Taking $k_{1}=1$ in Equation 13 and using the expressions obtained in Appendix yields

$$
\begin{aligned}
\Gamma & =\frac{\gamma}{\ell \omega}=\frac{8\left[1-\cos \left(\pi \sigma M_{\mathrm{A}}\right)\right]\left[\zeta-\sigma^{2}\left(2-M_{\mathrm{A}}^{2}\right)^{2}\right]}{\pi \sigma^{2}(\zeta-1)\left(2-M_{\mathrm{A}}^{2}\right)^{3}\left(4-\sigma^{2} M_{\mathrm{A}}^{2}\right)} \\
& \times\left[\frac{\left(2-\sigma^{2} M_{\mathrm{A}}^{2}\right)\left(M_{\mathrm{A}}^{2}-1+4 \sigma^{2}\right)}{\sigma^{2} M_{\mathrm{A}}^{2}\left(4-\sigma^{2} M_{\mathrm{A}}^{2}\right)}-1\right],
\end{aligned}
$$

where

$$
\sigma=\frac{\sqrt{\zeta}}{\sqrt{2(\zeta+1)-M_{\mathrm{A}}^{2}}}
$$

It is straightforward to show that $\Gamma>0$ in complete agreement with the previous discussion. When there is no flow, that is $M_{\mathrm{A}}=0$, this expression reduces to

$$
\Gamma=\frac{\pi(\zeta-1)}{8(\zeta+1)} .
$$

This expression coincides with the one obtained previously (see, e.g., Goossens, Erdélyi, and Ruderman, 2011). The dependence of $\Gamma$ on $M_{\mathrm{A}}$ is shown in Figure 1 for a few values of $\zeta$. We see that the decrement increases when $M_{\mathrm{A}}$ increases. It also increases with the increase of $\zeta$.

It is necessary to comment on the dependence of $\Gamma$ on $M_{\mathrm{A}}$. It looks as though the function $\Gamma\left(M_{\mathrm{A}}\right)$ has a discontinuity at $M_{\mathrm{A}}=M_{0}$ because $\Gamma=0$ for $M_{\mathrm{A}}>M_{0}$. In fact, the expression for $\Gamma$ given by Equation 22 is not valid when $M_{\mathrm{A}}$ is very close to $M_{0}$. The reason is that, formally, the resonant surface coincides with the internal boundary of the transitional layer when $M_{\mathrm{A}}=M_{0}$. The solution in the dissipative layer surrounding the ideal resonant surface is only valid when the distance between the resonant surface and the internal boundary of the transitional layer is larger than the thickness of the dissipative layer. When this distance decreases below the thickness of the dissipative layer the decrement starts to decrease sharply and becomes zero when the two surfaces coincide.

\subsection{Resonant Instability of Kink Oscillations}

Now we assume that Equation 18 is satisfied. As we have pointed out earlier, now it is possible that there are a few resonant surfaces. In Equation 13 the only restriction imposed on $k_{1}$ is $k_{1}<\sqrt{\zeta}$. The position of the $k$ th resonant surface is determined by $r=r_{k}$, where $r_{k}$ is defined by

$$
k \omega_{\mathrm{A}}\left(r_{k}\right)=\omega .
$$


Figure 1 Dependence of $\Gamma$ on $M_{\mathrm{A}} / M_{0}$ given by Equation 22 . The solid, dotted, and dashed curves correspond to $\zeta=3,10$, and 100 , respectively.

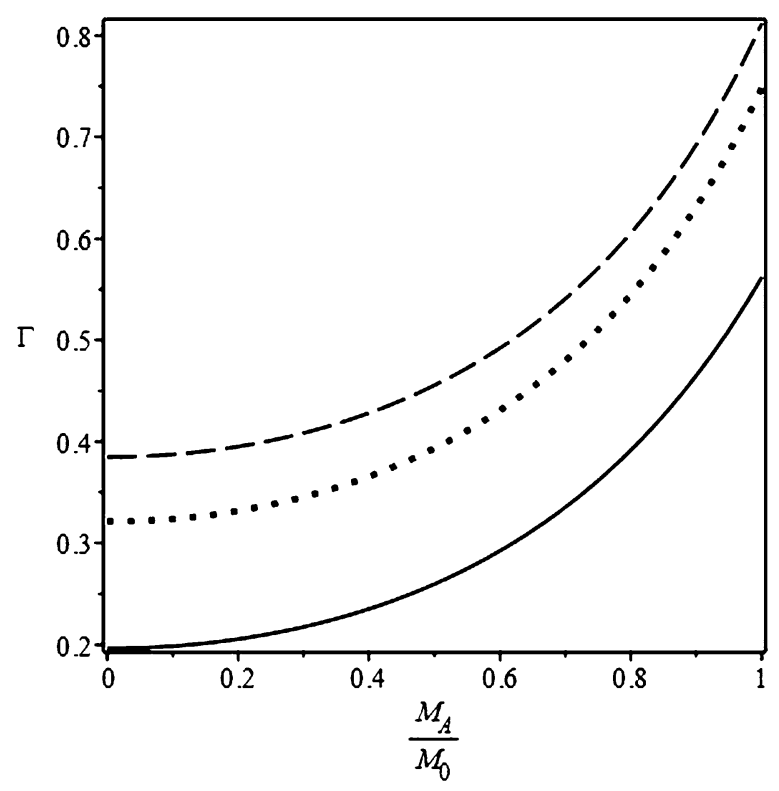

Using the expressions obtained in Appendix we obtain from Equation 13

$$
\begin{aligned}
\Gamma & =\frac{8 \sigma^{2} M_{\mathrm{A}}^{2}}{\pi \zeta(\zeta-1)\left(M_{\mathrm{A}}^{2}-2\right)^{2}} \sum_{k=1}^{k_{1}} k\left[\left(M_{\mathrm{A}}^{2}-\zeta-3\right)\right. \\
& \left.\times\left(\sigma^{2} M_{\mathrm{A}}^{2}-k^{2}-1\right)-4(\zeta+1)\right] \\
& \times \frac{\left[k^{2} \zeta-\sigma^{2}\left(M_{\mathrm{A}}^{2}-2\right)^{2}\right]\left[1+(-1)^{k} \cos \left(\pi \sigma M_{\mathrm{A}}\right)\right]}{\left[\sigma^{2} M_{\mathrm{A}}^{2}-(k-1)^{2}\right]^{2}\left[\sigma^{2} M_{\mathrm{A}}^{2}-(k+1)^{2}\right]^{2}} .
\end{aligned}
$$

It is shown in Appendix that the first term in the sum in this expression is negative. This implies that $\Gamma<0$ for $M_{1}<M_{\mathrm{A}}<M_{\mathrm{L}}$ when $k_{1}=1$ for all values of $M_{\mathrm{A}}<M_{\mathrm{L}}$, or $M_{1}<$ $M_{\mathrm{A}}<M_{2}$ when $M_{2}<M_{\mathrm{L}}$. Consequently, there is a resonant instability for these values of $M_{\mathrm{A}}$. We can see this in Figure 2. $|\Gamma|$ takes its maximum when $M_{\mathrm{A}}=M_{1}$, and it follows from Figure 2 that this maximum is almost independent of $\zeta$. On the other hand, $\Gamma=0$ for $M_{\mathrm{A}}=M_{1}$, so that the function $\Gamma\left(M_{1}\right)$ is discontinuous at $M_{\mathrm{A}}=M_{1}$. However, when $M_{\mathrm{A}}$ tends to $M_{1}$ from larger values, the resonant surface moves towards the internal boundary of the transitional layer. Equation 26 is not valid when the distance between the two surfaces is comparable with the thickness of the dissipative layer. In fact, there is a fast but continuous transition from the maximum value of $|\Gamma|$ to zero when $M_{1}$ varies in a small interval near $M_{1}$.

As we have already pointed out, the number of terms in the sum in Equation 26 depends on the value of $M_{\mathrm{A}}$, but it always less than $\sqrt{\zeta}$. Since the left panel of Figure 2 corresponds to $\zeta=3$ it follows from the inequality $k_{1}<\sqrt{\zeta}$ that the sum in Equation 26 contains only one term. As a result, the dependence of $\Gamma$ on $M_{\mathrm{A}}$ is continuous on the whole interval $M_{1}<M_{\mathrm{A}}<M_{\mathrm{L}}$. We also note that the increment of resonant instability decreases when $M_{\mathrm{A}}$ increases.

The middle panel in Figure 2 corresponds to $\zeta=10$, so that $k_{1} \leq 3$. The dependence of $\Gamma$ on $M_{\mathrm{A}}$ is discontinuous at $M_{\mathrm{A}}=M_{2}$ and $M_{\mathrm{A}}=M_{3}$. However, Equation 26 is not valid 

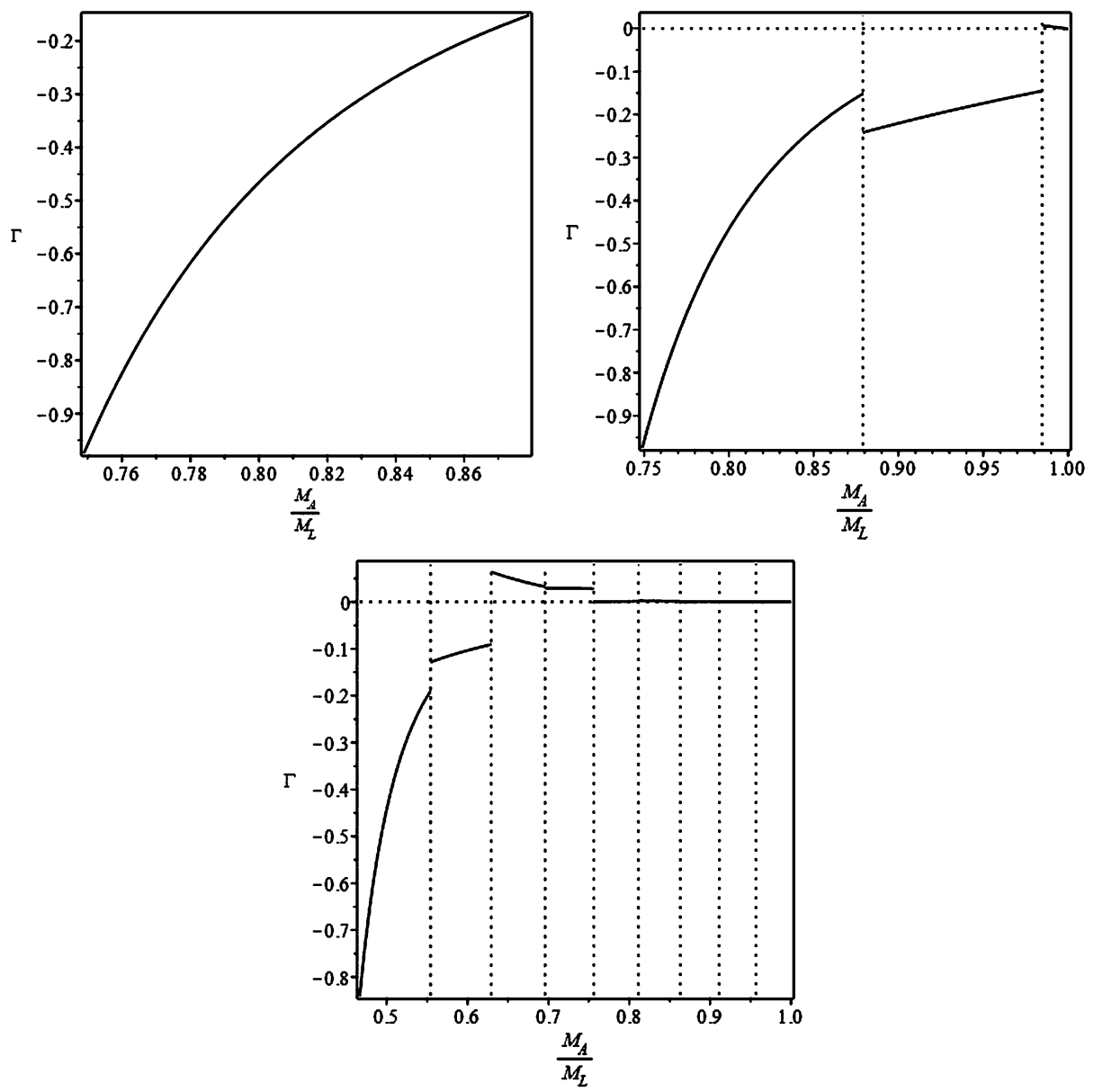

Figure 2 Dependence of $\Gamma$ on $M_{\mathrm{A}} / M_{\mathrm{L}}$ given by Equation 26. The upper-left, upper-right, and lower panels correspond to $\zeta=3,10$, and 100, respectively. In accordance with Equations 9 and 18 each curve is shown in the interval $\left[M_{1} / M_{\mathrm{L}}, 1\right]$. The vertical dotted lines in the upper-right panel correspond to $M_{\mathrm{A}}=M_{2}$ and $M_{\mathrm{A}}=M_{3}$, while in the lower-right panel they correspond to $M_{\mathrm{A}}=M_{2}, \ldots M_{\mathrm{A}}=M_{9}$, where the quantities $M_{k}, k>1$, are defined by equation 19 .

for $M_{\mathrm{A}}$ close to $M_{2}$ and $M_{3}$ because the second resonant surface coincides with the internal boundary of the transitional layer when $M_{\mathrm{A}}=M_{2}$, and the third resonant surface coincides with this boundary when $M_{\mathrm{A}}=M_{3}$. We note that the increment is a decreasing function of $M_{\mathrm{A}}$ in the intervals $\left(M_{1}, M_{2}\right)$ and $\left(M_{2}, M_{3}\right)$. We also see that the appearance of the second resonant surface when $M_{\mathrm{A}}$ moves from the first interval to the second results in the increase of the increment. Finally, we note that $\Gamma>0$ when $M_{\mathrm{A}} \in\left(M_{3}, M_{\mathrm{L}}\right)$. A propagating negativeenergy wave always grows when there is an energy sink. In the case of a standing wave, the situation is much more complex. It is a superposition of positive- and negative-energy waves. Whether the amplitude of a standing wave grows or decays depends on which of the two processes dominates: the amplification of the negative-energy wave or the damping of the positive-energy wave. We see that in the case when the energy sink is provided by resonant absorption both scenarios are possible. 
Finally, let us turn to the upper-right panel of Figure 2. It corresponds to $\zeta=100$. Since $\sqrt{\zeta}=10$ it follows that the sum in Equation 26 can contain up to nine terms. When $M_{\mathrm{A}}$ passes through $M_{k}, k=1, \ldots, 9$, a new resonant surface appears in the transitional layer. Again $|\Gamma|$ monotonically decreases in the intervals $\left(M_{1}, M_{2}\right)$ and $\left(M_{2}, M_{3}\right)$. However, now the appearance of the second resonant surface decreases the increment, and the appearance of the third resonant surface makes $\Gamma$ positive, so that the wave damps. We recall that $\Gamma>0$ corresponds to wave damping, and $\Gamma<0$ to wave growth. The wave damps for $M_{\mathrm{A}}>M_{3}$; however, the decrement is very small when $M_{\mathrm{A}}>M_{5}$.

\section{Application to Prominence Threads}

In this section we consider the possibility of excitation of kink oscillations of prominence threads by resonant instability. Flows are ubiquitously observed in prominences. Typical values of the flow speed in quiescent prominences do not exceed $35 \mathrm{~km} \mathrm{~s}^{-1}$ (Labrosse et al., 2010; Mackay et al., 2010; Arregui, Oliver, and Ballester, 2018); however, they can be as high as $200 \mathrm{~km} \mathrm{~s}^{-1}$ in active-region prominences. The magnetic-field magnitude also can vary by more than an order of magnitude, from $3 \mathrm{G}$ to $45 \mathrm{G}$, while the typical neutral hydrogen number density is $3 \times 10^{16} \mathrm{~m}^{-3}$ (Labrosse et al., 2010). This implies that the Alfvén speed can vary from as low as $30 \mathrm{~km} \mathrm{~s}^{-1}$ to as high as $500 \mathrm{~km} \mathrm{~s}^{-1}$. In prominence threads $\zeta \gg 1$, so that $M_{1} \approx 1.85$. Hence, in accordance with the results obtained in the previous section, prominence threads can be resonantly unstable if the flow speed is larger that 1.85 times the Alfvén speed in the thread. We see that the resonant instability threshold velocity is approximately $55 \mathrm{~km} \mathrm{~s}^{-1}$ when the Alfvén speed is $30 \mathrm{~km} \mathrm{~s}^{-1}$, and it is approximately $185 \mathrm{~km} \mathrm{~s}^{-1}$ when the Alfvén speed is $100 \mathrm{~km} \mathrm{~s}^{-1}$. Hence, the resonant-flow instability must be considered as a possible mechanism of excitation of prominence-thread kink oscillations. We recall that the $\mathrm{KH}$ instability threshold is $\sqrt{2(\zeta+1)}$ times the Alfvén speed, so for $\zeta=100$ it is approximately $420 \mathrm{~km} \mathrm{~s}^{-1}$ even for the lowest possible value of Alfvén speed equal to $30 \mathrm{~km} \mathrm{~s}^{-1}$. Hence, the prominence-thread kink oscillations definitely cannot be excited by the KH instability.

Another interesting phenomenon that can appear in a prominence thread is the existence of undamped oscillations. As was shown in Section 4, the amplitude of kink oscillations does not change when $M_{0}<M_{\mathrm{A}}<M_{1}$. We obtain $M_{0} \approx 0.765$ for $\zeta \gg 1$.

\section{Summary and Conclusion}

In this article we studied kink oscillations of a magnetic tube with siphon flow. We used the cold-plasma approximation and assumed that the background magnetic field everywhere has constant magnitude and is parallel to the tube axis. Siphon flow has constant velocity parallel to the magnetic field, and it is confined to the tube core. The tube consists of the core and transitional or boundary layer where the plasma density decreases from its value in the tube core to that in the surrounding plasma. We used the thin-tube and thin-boundary (TTTB) approximation. To study the evolution of kink oscillations we used the expression for the decrement/increment $\gamma$ derived by Ruderman and Petrukhin (2019).

The decrement/increment $[\gamma]$ is proportional to the relative thickness of the transitional layer $[\ell]$, which is the ratio of the thickness of transitional layer to the tube radius. We introduced the dimensional quantity $\Gamma$ that is the ratio of $\gamma$ to $\ell$ times the oscillation frequency. This quantity depends on two dimensionless parameters: $\zeta$, which is the density contrast, 
and the Mach number $M_{\mathrm{A}}$, which is the ratio of the flow speed to the Alfvén speed in the tube core.

In our analysis we concentrated on the fundamental mode of kink oscillations. We only considered non-leaky oscillations with the oscillation frequencies below the fundamental Alfvén frequency in the external plasma. This condition is written as $M_{\mathrm{A}}<M_{\mathrm{L}}$, where $M_{\mathrm{L}}$ is defined by Equation 9 . We proved that $\Gamma>0$ when $M_{\mathrm{A}}<M_{0}$ where $M_{0}$ is defined by Equation 17. This result implies that the fundamental kink mode damps due to resonant absorption when $M_{\mathrm{A}}<M_{0}$. Next, we showed that $\Gamma=0$ when $M_{0}<M_{\mathrm{A}}<M_{1}$, where $M_{1}$ is defined by Equation 18. Hence, the fundamental kink mode does not either damp or grow when $M_{0}<M_{\mathrm{A}}<M_{1}$.

The standing kink mode is a superposition of two propagating kink modes. One of them becomes a negative-energy wave when $M_{\mathrm{A}}>\sqrt{2}$. Then the kink oscillation can be unstable. However, to make this oscillation growing one needs to have an energy sink. In the problem studied in this article, the energy sink is provided by resonant absorption. It only operates when the oscillation frequency is in the Alfvén continuum. To satisfy this condition we need $M_{\mathrm{A}}>M_{1}>\sqrt{2}$. The Alfvén continuum is the union of an infinite number of intervals corresponding to the fundamental mode of local Alfvén frequency as well as all of its overtones. This implies that, in principle, it is possible that the condition of resonance is satisfied in a few spatial positions in the transitional layer, that is, there can be a few resonant surfaces. The number of resonant surfaces depends on the value of $M_{\mathrm{A}}$, but it is less than $\sqrt{\zeta}$.

When $M_{\mathrm{A}}>M_{1}$ one of the two waves constituting the standing wave should grow due to resonant absorption, while the positive-energy wave should decay. Since both waves must either grow or decay simultaneously, the standing wave either grows or damps depending of which of the two processes, the negative-wave growth or positive-energy wave decay, dominates. The examination of Figure 2 show that both scenarios are possible.

We applied the theoretical results to the problem of excitation of kink oscillations in prominence threads. We showed that, for particular values of thread parameters, kink oscillations of prominence threads can be excited by resonant instability related to the presence of siphon flows.

Finally, we make a brief comment on the approximations used in this article. The first one is the zero- $\beta$ approximation. This approximation is sufficiently accurate for kink waves both in coronal magnetic loops as well as prominence threads. In addition, in the thin-tube approximation the finite plasma pressure does not affect the properties of kink waves. This is because in a thin tube a kink wave practically does not perturb the plasma density and pressure. The second is the thin-tube approximation. We expect that this approximation should work very well because the radii of coronal loops and prominence threads are much smaller than their lengths. The last approximation is the thin-boundary-layer approximation. At first sight using this approximation looks doubtful. Actually, it seems more realistic that the density varies either through the whole loop cross-section or at least through a substantial part. This problem has been addressed by a few authors. Tatsuno and Wakatani (1998) studied the resonant damping of surface waves in a magnetic slab. They found that the thin-boundarylayer approximation works very well for $\ell \lesssim 0.2$ For larger values of $\ell$ the dependence of the damping rate on $\ell$ is not monotonic. Van Doorsselaere et al. (2004) and Soler et al. (2013) considered the same problem for magnetic tubes. Again they found that the dependence of decrement on $\ell$ is not monotonic. However, the thin-tube approximation works better for magnetic tubes than for slabs. The analytically found decrement using the thin-tube approximation differs from that found in the direct numerical modelling by less than $10 \%$ for $\ell \lesssim 0.5$. We note that the thickness of the transitional layer is twice as big as the tube-core radius when $\ell=0.5$. We believe that the thin-tube approximation works equally well when calculating the increment of the resonant instability. 


\section{Appendix: Calculation of Decrement/Increment}

In this appendix we calculate various quantities present in Equation 13. We recall that $n=1$. Using Equations 8 and 10 yields

$$
\frac{4 \pi^{2} L^{2} \sqrt{\zeta}}{R V_{\mathrm{Ai}}^{2} \sqrt{2(\zeta+1) V_{\mathrm{Ai}}^{2}-U^{2}}}=\frac{4 \pi^{5} \sigma}{R L \omega_{\mathrm{Ai}}^{3}}
$$

Next, we use Equations 7, 8, 10, 12, 13, 20, and 21 to obtain

$$
\begin{gathered}
h=\frac{\pi \sigma M_{\mathrm{A}}}{L}, \quad \Delta_{k}=\frac{\omega_{\mathrm{Ai}}^{2} \sigma^{4}(\zeta-1)\left(M_{\mathrm{A}}^{2}-2\right)^{4}}{l R \zeta k^{2}}, \\
\frac{k h W_{k} V_{\mathrm{Ak}}^{2}}{\Delta_{k}}=\frac{k \ell R L M_{\mathrm{A}} \omega_{\mathrm{Ai}}^{2}\left[k^{2} \zeta-\sigma^{2}\left(M_{\mathrm{A}}^{2}-2\right)^{2}\right]}{\pi \sigma(\zeta-1)\left(M_{\mathrm{A}}^{2}-2\right)^{2}}, \\
\frac{h L C_{2}+\pi(n \pm k) C_{1}}{h^{2} L^{2}-\pi^{2}(n \pm k)^{2}}=\frac{\omega_{\mathrm{Ai}}^{2} \sigma M_{\mathrm{A}}\left[\sigma^{2}\left(M_{\mathrm{A}}^{2}-4\right) \mp 2 k-1-M_{\mathrm{A}}^{2}\right]}{\pi\left[\sigma^{2} M_{\mathrm{A}}^{2}-(k \pm 1)^{2}\right]} \\
\frac{1-(-1)^{n+k} \cos (h L)}{\left[h^{2} L^{2}-\pi^{2}(n-k)^{2}\right]\left[h^{2} L^{2}-\pi^{2}(n+k)^{2}\right]} \\
=\frac{1+(-1)^{k} \cos \left(\pi \sigma M_{\mathrm{A}}\right)}{\pi^{4}\left[\sigma^{2} M_{\mathrm{A}}^{2}-(k-1)^{2}\right]\left[\sigma^{2} M_{\mathrm{A}}^{2}-(k+1)^{2}\right]} .
\end{gathered}
$$

Using Equations 23 and 30 we obtain

$$
\begin{aligned}
& \frac{h L C_{2}+\pi(n-k) C_{1}}{h^{2} L^{2}-\pi^{2}(n-k)^{2}}+\frac{h L C_{2}+\pi(n+k) C_{1}}{h^{2} L^{2}-\pi^{2}(n+k)^{2}} \\
& =\frac{2 \sigma M_{\mathrm{A}} \omega_{\mathrm{Ai}}^{2}}{\pi\left[\sigma^{2} M_{\mathrm{A}}^{2}-(k-1)^{2}\right]\left[\sigma^{2} M_{\mathrm{A}}^{2}-(k+1)^{2}\right]} \\
& \quad \times\left\{\left(\sigma^{2} M_{\mathrm{A}}^{2}-k^{2}-1\right)\left[\sigma^{2}\left(M_{\mathrm{A}}^{2}-4\right)-1-M_{\mathrm{A}}^{2}\right]-4 k^{2}\right\} \\
& =\frac{2 \sigma^{3} M_{\mathrm{A}} \omega_{\mathrm{Ai}}^{2}\left(M_{\mathrm{A}}^{2}-2\right)}{\pi \zeta\left[\sigma^{2} M_{\mathrm{A}}^{2}-(k-1)^{2}\right]\left[\sigma^{2} M_{\mathrm{A}}^{2}-(k+1)^{2}\right]} \\
& \quad \times\left[\left(\sigma^{2} M_{\mathrm{A}}^{2}-k^{2}-1\right)\left(M_{\mathrm{A}}^{2}-\zeta-3\right)-4(\zeta+1)\right] .
\end{aligned}
$$

Now we prove that the first term in Equation 26 is negative. We take $k=1$ and write

$$
\left(M_{\mathrm{A}}^{2}-\zeta-3\right)\left(\sigma^{2} M_{\mathrm{A}}^{2}-2\right)-4(\zeta+1)=\frac{F\left(M_{\mathrm{A}}^{2}\right)}{2(\zeta+1)-M_{\mathrm{A}}^{2}},
$$

where

$$
F\left(M_{\mathrm{A}}^{2}\right)=(\zeta+2) M_{\mathrm{A}}^{4}-(\zeta+2)(\zeta+3) M_{\mathrm{A}}^{2}-4\left(\zeta^{2}-1\right)
$$

We prove that $F\left(M_{\mathrm{A}}^{2}\right)<0$ for $M_{1}<M_{\mathrm{A}}<M_{\mathrm{L}}$ when $k_{1}=1$, and for $M_{1}<M_{\mathrm{A}}<M_{2}$ when $k_{1}>1$. Since the roots of $F\left(M_{\mathrm{A}}^{2}\right)$ considered as a quadratic polynomial of $M_{A}^{2}$ have different signs it is enough to prove that $F\left(M_{\mathrm{L}}^{2}\right)<0$ when $k_{1}=1$ and $F\left(M_{2}^{2}\right)<0$ when $k_{1}>1$. 
First we consider the case where $k_{1}=1$. In this case

$$
2 F\left(M_{\mathrm{L}}^{2}\right)=-\zeta[(\zeta+1) \sqrt{8 \zeta+1}+7 \zeta+2] .
$$

When $k_{1}>1$ we obtain

$$
\begin{aligned}
\zeta^{2} F\left(M_{2}^{2}\right) & =-\zeta^{2}\left(6 \zeta^{2}-4 \zeta-2\right)+16 \zeta^{2}+4 \zeta+16 \\
& -2\left(\zeta^{3}+\zeta^{2}+2 \zeta+8\right) \sqrt{2 \zeta^{2}+1}
\end{aligned}
$$

We prove that

$$
\left(\zeta^{3}+\zeta^{2}+2 \zeta+8\right) \sqrt{2 \zeta^{2}+1}>2\left(4 \zeta^{2}+\zeta+4\right) .
$$

Squaring the two parts of this inequality we see that it is equivalent to an obvious inequality

$$
2 \zeta^{6}+4 \zeta^{5}+11 \zeta^{4}+42 \zeta^{3}-19 \zeta^{2}+52 \zeta+16>0 .
$$

It follows from Equations 36 and 37 that $F\left(M_{2}^{2}\right)<0$. Next, we obtain

$$
\begin{aligned}
m^{2} \zeta & -\sigma^{2}\left(M_{\mathrm{A}}^{2}-2\right)^{2}=\zeta\left(1-\frac{\left(M_{\mathrm{A}}^{2}-2\right)^{2}}{2(\zeta+1)-M_{\mathrm{A}}^{2}}\right) \\
& >\zeta\left(1-\frac{\left(M_{\mathrm{L}}^{2}-2\right)^{2}}{2(\zeta+1)-M_{\mathrm{L}}^{2}}\right)=0 .
\end{aligned}
$$

It follows from Equation 39 and the inequality $F\left(M_{\mathrm{A}}^{2}\right)<0$ that the first term in the sum in Equation 26 is negative.

Acknowledgements The authors gratefully acknowledge financial support from the Russian Fund for Fundamental Research (RFFR) grant (19-02-00111).

\section{Declarations}

Disclosure of Potential Conflicts of Interest The authors declare that they have no conflicts of interest.

Open Access This article is licensed under a Creative Commons Attribution 4.0 International License, which permits use, sharing, adaptation, distribution and reproduction in any medium or format, as long as you give appropriate credit to the original author(s) and the source, provide a link to the Creative Commons licence, and indicate if changes were made. The images or other third party material in this article are included in the article's Creative Commons licence, unless indicated otherwise in a credit line to the material. If material is not included in the article's Creative Commons licence and your intended use is not permitted by statutory regulation or exceeds the permitted use, you will need to obtain permission directly from the copyright holder. To view a copy of this licence, visit http://creativecommons.org/licenses/by/4.0/.

\section{References}

Abedini, A.: 2018, Solar Phys. 293, 22. DOI.

Andries, J., Goossens, M.: 2001, Astron. Astrophys. 368, 1083. DOI.

Andries, J., Tirry, W.J., Goossens, M.: 2000, Astrophys. J. 531, 561. DOI.

Arregui, I., Oliver, R., Ballester, J.L.: 2018, Liv. Rev. Solar Phys. 15, 3. DOI.

Aschwanden, M.J., Fletcher, L., Schrijver, C.J., Alexander, D.: 1999, Astrophys. J. 520, 880. DOI.

Bahari, K., Petrukhin, N.S., Ruderman, M.S.: 2020, Mon. Not. Roy. Astron. Soc. 496, 67. DOI.

Benjamin, T.B.: 1963, J. Fluid Mech. 16, 436. DOI.

Brekke, P., Kjeldseth-Moe, O., Harrison, R.A.: 1997, Solar Phys. 175, 511. DOI. 
Cairns, R.A.: 1979, J. Fluid Mech. 92, 1. DOI.

Chae, J., Ahn, K., Lim, E.-K., Choe, G.S., Sakurai, T.: 2008, Astrophys. J. Lett. 689, L73. DOI.

Doyle, J.G., Taroyan, Y., Ishak, B., Madjarska, M.S., Bradshaw, S.J.: 2006, Astron. Astrophys. 452, 1075. DOI.

Duckenfield, T., Anfinogentov, S.A., Pascoe, D.J., Nakariakov, V.M.: 2018, Astrophys. J. Lett. 854, L5. DOI.

Erdélyi, R., Taroyan, Y.: 2003, J. Geophys. Res. 108, 1043. DOI.

Erdélyi, R., Taroyan, Y.: 2008, Astron. Astrophys. 489, L49. DOI.

Fabrikant, A.L., Stepanyants, Y.: 1998, Propagation of Waves in Shear Flows, World Scientific Series on Nonlinear Science Series A 18, World Scientific, Singapore.

Goossens, M., Andries, J., Aschwanden, M.J.: 2002, Astron. Astrophys. 394, L39. DOI.

Goossens, M., Erdélyi, R., Ruderman, M.S.: 2011, Space Sci. Rev. 158, 289. DOI.

Goossens, M., Hollweg, J.V., Sakurai, T.: 1992, Solar Phys. 138, 233. DOI.

Goossens, M., Terradas, J., Andries, J., Arregui, I., Ballester, J.L.: 2009, Astron. Astrophys. 503, 213. DOI.

Gruszecki, M., Nakariakov, V.M., Van Doorsselaere, T., Arber, T.D.: 2010, Phys. Rev. Lett. 105, 055004. DOI.

Hollweg, J.V., Yang, G.: 1988, J. Geophys. Res. 93, 5423. DOI.

Ionson, N.H.: 1978, Astrophys. J. 226, 650. DOI.

Joarder, P.S., Nakariakov, V.M., Poberts, B.: 1997, Solar Phys. 176, 285. DOI.

Kadomtsev, B.B., Mikhailovskii, A.V., Timofeev, A.V.: 1965, Sov. Phys. JETP 20, 1517.

Kohutova, P., Verwichte, E.: 2017, Astron. Astrophys. 606, A120. DOI.

Labrosse, N., Heinzel, P., Vial, J.C., Kucera, T., Parenti, S., Gunár, S., Schmieder, B., Kilper, G.: 2010, Space Sci. Rev. 151, 243. DOI.

Mackay, D.H., Karpen, J.T., Ballester, J.L., Schmieder, B., Aulanier, G.: 2010, Space Sci. Rev. 151, 333. DOI.

Mikhailovskii, A.V.: 1974, Theory of Plasma Instabilities, Consultants Bureau, Washington.

Nakariakov, V.M., Ofman, L., DeLuca, E.E., Roberts, B., Davila, J.M.: 1999, Science 285, 862. DOI.

Nechaeva, A., Zimovets, I.V., Nakariakov, V.M., Goddard, C.R.: 2019, Astrophys. J. Suppl. Ser. 241, 31 . DOI.

Nezlin, M.V.: 1976, Sov. Phys. Usp. 19, 946. DOI.

Ofman, L.A., Wang, T.J.: 2008, Astron. Astrophys. 482, L9. DOI.

Ostrovskii, L.A., Rybak, S.A., Tsimring, L.S.: 1986, Sov. Phys. Usp. 29, 1040. DOI.

Pierce, J.: 1974, Almost All About Waves, MIT Press, Cambridge.

Ruderman, M.S.: 2010, Solar Phys. 267, 377. DOI.

Ruderman, M.S.: 2011, Solar Phys. 271, 41. DOI.

Ruderman, M.S.: 2018, J. Plasma Phys. 84, 905840101. DOI.

Ruderman, M.S., Belov, N.A.: 2010, In: Bar-Yoseph, P.Z., Brøns, M., Cliffe, K.A., Gelfgat, A., Oron, A. (eds.) Third International Symposium on Bifurcations and Instabilities in Fluid Dynamics, J. Phys. CS216, 012016. DOI.

Ruderman, M.S., Goossens, M.: 1995, J. Plasma Phys. 54, 149. DOI.

Ruderman, M.S., Petrukhin, N.S.: 2019, Astron. Astrophys. 631, A31. DOI.

Ruderman, M.S., Roberts, B.: 2002, Astrophys. J. 577, 475. DOI.

Ruderman, M.S., Shukhobodskiy, A.A., Erdélyi, R.: 2017, Astron. Astrophys. 602, A50. DOI.

Ruderman, M.S., Wright, A.N.: 1998, J. Geophys. Res. 103, 26573. DOI.

Ryutova, M.P.: 1988, Sov. Phys. JETP 67, 1594.

Soler, R., Goossens, M., Terradas, J., Oliver, R.: 2013, Astrophys. J. 777, 158. DOI.

Stepanyants, Y., Fabrikant, A.L.: 1989, Sov. Phys. Usp. 32, 783. DOI.

Su, W., Guo, Y., Erdélyi, R., Ning, Z.J., Ding, M.D., Cheng, X., Tan, B.L.: 2018, Sci. Rep. 8, 4471. DOI.

Taroyan, Y., Ruderman, M.S.: 2011, Space Sci. Rev. 158, 505. DOI.

Tatsuno, T., Wakatani, M.: 1998, J. Phys. Soc. Japan 67, 2322. DOI.

Teriaca, L., Banerjee, D., Falchi, A., Doyle, J.G., Madjarska, M.S.: 2004, Astrophys. J. 427, 1065. DOI.

Terradas, J., Arregui, I., Oliver, R., Ballester, J.L.: 2008, Astrophys. J. Lett. 678, L153. DOI.

Tian, H., Curdt, W., Marsch, E., He, J.: 2008, Astrophys. J. Lett. 681, L121. DOI.

Tian, H., Marsch, E., Curdt, W., He, J.: 2009, Astrophys. J. 704, 883. DOI.

Van Doorsselaere, T., Andries, J., Poedts, S., Goossens, M.: 2004, Astrophys. J. 606, 1223. DOI.

Winebarger, A.R., DeLuca, E.E., Golub, L.: 2001, Astrophys. J. Lett. 553, L81. DOI.

Winebarger, A.R., Warren, H., van Ballegooijen, A., DeLuca, E.E., Golub, L.: 2002, Astrophys. J. Lett. 567, L89. DOI.

Zaqarashvili, T.V., Vörös, Z., Zhelyazkov, I.: 2014, Astron. Astrophys. 561, A62. DOI.

Zhelyazkov, I.: 2012, Astron. Astrophys. 537, A124. DOI.

Zhelyazkov, I., Zaqarashvili, T.V.: 2012, Astron. Astrophys. 547, A14. DOI.

Zhelyazkov, I., Zaqarashvili, T.V., Chandra, R., Srivastava, A.K., Mishonov, T.: 2015, Adv. Space Res. 56, 2727. DOI.

Zimovets, I.V., Nakariakov, V.M.: 2015, Astron. Astrophys. 577, A4. DOI.

Publisher's Note Springer Nature remains neutral with regard to jurisdictional claims in published maps and institutional affiliations. 